\title{
Next-Generation Directional mmWave MAC Time-Spatial Resource Allocation
}

\author{
Chan-Yu Tung, Chun-Yen Chen, Hung-Yu Wei \\ Graduate Institute of Communication Engineering, National Taiwan University
}

\begin{abstract}
This paper will design a resource allocation algorithm, mainly on downlink transmission by Base Station (BS) to User Equipments (UEs) implemented using directional antenna. The resource allocation is accomplished by Pair-Wisely BottomUp (PWBU) approach, targeting a time-efficient allocation based on SINR feedbacks. We utilize adaptive smart antennas capable of simultaneous transmission toward different target device ends. The goal of allocation is to determine which UEs to serve in each time-slots. At the end of this paper, we will evaluate performance of PWBU heuristic allocation. The designed algorithm will achieve time-efficient and sub-optimal resource allocation compared to the results of exhaustive optimization.
\end{abstract}

Index Terms-Basic Switched Directional Antenna, Adaptive Antenna Array, Time Spatial Resource Allocation

\section{INTRODUCTION}

Directional beamforming enables resource allocation of spaces and increased signal strength, toward desired deviceend. By utilizing spatial resources, we can minimize the amount of interferences, while more users can communicate under identical frequency and time [1]. To study simultaneous data transmission by adaptive antenna array, we allow simultaneous and non-identical data transmission at the same time to different device-ends. In this paper we assume Base Stations(BS) have this beamforming capability. Previous utilization of such technology is applied on ad-hoc networks in [2-5].

We develop a Pair-Wisely Bottom-Up (PWBU) algorithm for the BS to allocate UEs into time and spatial dimensions, while maximizing system throughput. Evaluation will compare throughput to the optimum value calculated by exhaustive approach and Time-Division Multiple Access (TDMA) method. We assume Simultaneous service is done by transmitting to more than one UE by equally shared power. On the other hand, in TDMA all UEs are served in individual slots with full power. In both cases, the total power is a fixed value. To ease the throughput measurement, we adopt full buffer traffic model for all UEs.

The remaining part of this paper is organized as follows. Section II discusses related works prior to directional resource allocation. Section III illustrates the system model. Section IV focuses the procedure to allocate time-spatial resources. Section V will evaluate the PWBU algorithm. Section VI will recapitulate the paper.

\section{RELATED RESEARCHES}

The inspiration to design PWBU algorithm is the forseen

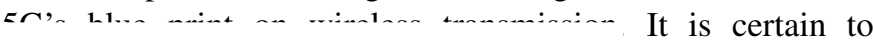

develop on mmWave frequency [6]. Experimental evaluations have shown feasibility on outdoor by LoS and NLoS transmission scenarios [7, 8]. As outdoor mmWave official standard is ready, the future mobile capacity foresees tremendous amount of increase [1]. To best of our belief, directional MAC resource allocation is a potential field to research on.

There are many papers discussing about the MAC issues of directional wireless network scenario, but most of them are under the $802.15 .3 \mathrm{c}$ or 802.11 ad environment, and are all adhoc situation[9-14]. The main difference is that $802.11 \mathrm{ad}$ or 802.15.3c scenarios mainly handles numerous point-to-point connections while cellular BS will have to allocate many number of UEs at the same time. An overview of directional antenna and its mac layer issues are well described in [11], but also mainly relate to ad-hoc situation. In [12][13], the 802.11ad AP acts as a coordinator and group non-interfering transmissions. For WPAN [10] introduced a concept of $E x$ clusive Region to define the concurrent transmission region. Cellular BS can deal with interferences between transmissions with more information, as a result, the solution should be quite different, and we haven't found papers that talk about resource allocation for directional BS specific for cellular network.

Due to ongoing discussion of $5 \mathrm{G}$ mmWave, this paper will implement on IEEE TGad standardized channel. Based on document [15], mainly for simulation of performance evaluation. Preliminary research documents have been released indicating the feasibility for fast data streaming with 802.11 AD [16]. We select $802.11 \mathrm{AD}$ as an alternative to $5 \mathrm{G}$ channels. Targeting to ease the antenna complexity, transmission will adapt basic and switched antenna patterns, based on preliminary analysed models [11, 15].

\section{System ModeL}

This section will focus on the explanations of utilized devices, antenna, and channel models for the paper.

\section{A. Transmission Devices}

In this paper we only consider single BS with multiple UEs. Set $\bar{N}_{0}$ are UEs associated to the BS. Downlink transmission at mmWave are directional, equipped with the antenna model in Section III-B. The receiving pattern of UEs are omnidirectional, in other words, UE can hear signals from all directions. We assume each beam formed by the BS occupies the whole bandwidth, so frequency division multiple access is not considered. 


\section{B. Antenna Model}

To reduce the complexity from analysing the randomness of real antennas, this paper will utilize basic and switched directional antenna [15]. Basic antenna exhibits modelled behaviour of decaying gain at main-lobe, or direction of transmission, and takes averaged gain value outside at the sidelobe. Switched antenna can only transmit in discrete direction in which the values are not continuous.

Basic antennas are like real antennas with width of the mainlobe defined as Half Power Beamwidth $\theta_{B}$. The point where antenna gain falls by $3 \mathrm{~dB}$ from the center. Fig. 1 shows $\theta_{B}$ values of $15^{\circ}, 30^{\circ}$, and $60^{\circ}$. The curved line is the main-lobe and flat line is side-lobe. We use $15^{\circ}$ for $\theta_{B}$.

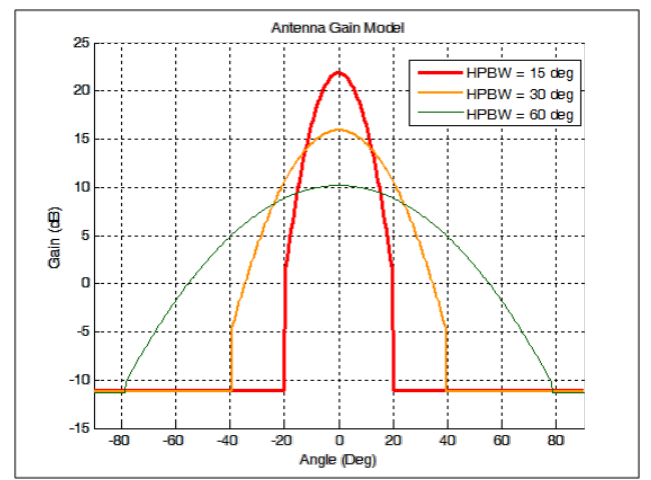

Fig. 1: Graph illustrating example gains of directional antenna

Equation (1) is antenna gain in decibels. The signal gain is function of deviated direction $\phi$. Observe deviating from center away by an angle value of $\frac{\theta_{B}}{2}$ reduces $G_{t}$ by $3 \mathrm{~dB}$.

$$
G_{t}(\phi)= \begin{cases}G_{0, d B}-12\left(\frac{\phi}{\theta_{B}}\right)^{2} & |\phi| \leq 1.3 \theta_{B} \\ G_{\aleph, d B} & |\phi|>1.3 \theta_{B}\end{cases}
$$

Maximum antenna gain $G_{0, d B}$ occurs when receiver is on direction of transmission. Gain outside main lobe with $20 \mathrm{~dB}$ drop from peak, becomes gain of $G_{\aleph, d B}$ with averaged gain values at side-lobe of real antennas. Value $k_{0}$ is antenna parameters and defines gain in Equation (2) and Equation (3).

$$
\begin{gathered}
G_{0, d B}=\left(\frac{k_{0}}{\sin \frac{\theta_{B}}{2}}\right)^{2} \\
G_{\aleph, d B}=-10.6-0.41 \log \theta_{B}
\end{gathered}
$$

We will model on switched antenna patterns [11], to distinguish direction of transmission. Allowable directional of beams are $\bar{A}$. We utilize a pattern $\bar{A}=\left\{0^{\circ}, 15^{\circ}, \cdots\right\}$. Transmission beam assigned by the BS to UE $i$, by which $i \in \bar{N}_{0}$, is defined as $\alpha_{i}$ and satisfies $\alpha_{i} \in \bar{A}$.

Simultaneous beamforming by adaptive array is achievable by sharing power. Each of the beam's power $p_{x}$ are same when added up, equal to value of single beam $p_{x, 0}$. To meet indicated requirement, Equation (4) represent transmitting power by BS's simultaneous beamforming.

$$
p_{x}(t)=\frac{p_{x, 0}}{|\bar{n}(t)|}
$$

UEs set assigned in one time-slot $t$ of equal duration is defined as $\bar{n}(t)$. Value $|\bar{n}(t)|$ defines total UEs in time-slot $t$. The more UEs are simultaneously served by BS at $t$, the less the power UEs receive (Fig. 2).

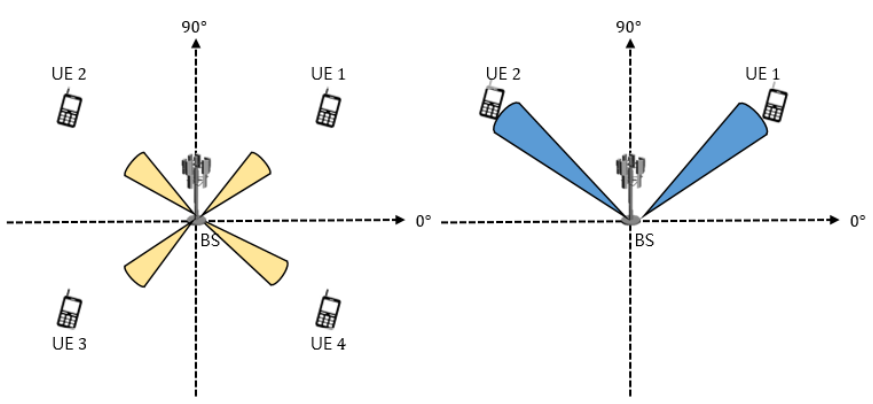

Fig. 2: Power distribution when different number of UEs are served in one time slot. On the left side 4 UEs are served simultaneously and the power is divided by 4 . On the right side only 2 UEs are served so the power is stronger.

\section{Channel Model}

This section will introduce how mmWave signals are calculated in this paper by considering directional factors.

Signal to Interference and Noise ratio (SINR) received by $\mathrm{UE} i$ at time $t$ is represented by Equation (5). Received channel thermal noise is $G_{W}$, contributed by bandwidth in $\mathrm{GHz}$ range.

$$
\gamma_{i}(t)=\frac{p_{x}(t) g_{i i}}{G_{W}+\sum_{j \neq i, j \in \bar{n}(t)} p_{x}(t) g_{i j}}
$$

Signal strength with channel attenuation received by UE $i$ is $p_{x}(t) g_{i i}$. Because several beam directions will be formed at the same time, each beam may cause interference to other signal beams. Assuming notation $j$ represents all other signal beams to other UEs except UE $i$, interference is $p_{x}(t) g_{i j}$. Channel signal and interference attenuation are $g_{i i}$ and $g_{i j}$.

Due to single BS as a originating source, SINR of a device $\mathrm{UE} i$ is function of distance $d_{i}$ to $\mathrm{BS}$, and with angle of UE $i$ 's signal beam to device $\phi_{i i}$ with angle of the other UE $j$ s' signal beams to the device $\phi_{i j}$.

Signal attenuation in logarithmic form is Eq. (6). Interference attenuation is Eq. (7). $G_{t}$ represents antenna gain from the BS and $L$ for path loss. Notice the assumption of omni-directional antenna at receiver end, and there will be no additional receiving signal gain.

$$
\begin{aligned}
& g_{i i}=G_{t}\left(\phi_{i i}\right)-L\left(d_{i}\right) \\
& g_{i j}=G_{t}\left(\phi_{i j}\right)-L\left(d_{i}\right)
\end{aligned}
$$

Attenuations can be calculated by distance with path-loss equation $L(d)$, antenna parameters, and transmitted power. The conference room environment channel model in [15] is adopted in our work. It considers NLoS rays including reflections that experience extra attenuation with additional 
distances from bouncing. Each of ray bounce suffers additional signal decay. Detailed explanations are upon official document and therefore neglected.

\section{PWBU Algorithm OF RESOURCE Allocation}

Pair-Wisely Bottom-up(PWBU) algorithm allocates resource by signal strength information of each UE repect to each switched beam direction. Bottom-up by the optimized beam results of paired UEs into multiple UE allocation. The outermost platform is shown in Function 1, requires input of associated UEs and returns allocated time-slot and corresponding beam direction.

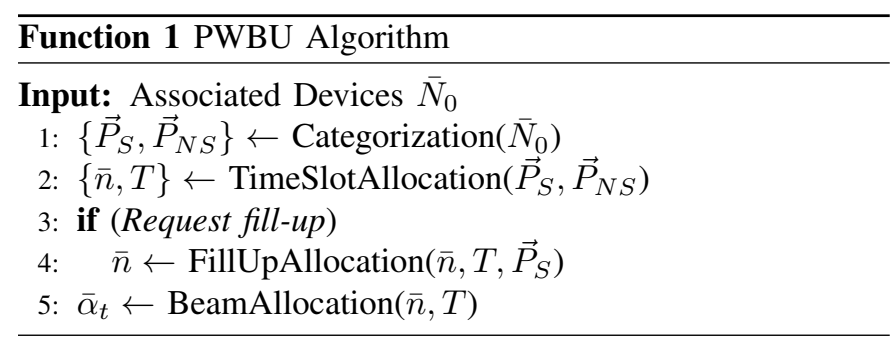

In the function, Line 1 categorizes associated devices $\bar{N}_{0}$ into pair by pair element vectors, and determines whether they are capable of being simultaneously served $\vec{P}_{S}$ or not $\vec{P}_{N S}$.

Line 2 utilizes the information of pair vectors and allocate UEs into time-slots $\bar{n}(t)$ in which $1 \leq t \leq T$. Time unit $T$ is the maximum time slots allocated, and is determined by TimeslotAllocation. In the function $T$ is decided once every UE has been served once. The allocation algorithm targets to increase throughput by reducing $T$.

Originally the allocation ends when all UEs are served once. However if fill-up is requested in Line 3, then UEs can fill into already allocated slots as long as it meat certain criteria (line 4). Requested fill-up will allow one or more UEs served more than once, and returns updated $\bar{n}$ without altering $T$.

Line 5 , is the last step that determines the beam direction $\bar{\alpha}$ for each UEs in each time-slots.

\section{A. Device Categorization}

Function categorization distinguishes each combination pairs by whether capable of being served simultaneously or not. The process is accomplished by PairAnalysis (Function 3), details in Section IV-B. PairAnalysis analyzes five beam direction combination and see if the paired UEs can be served in the same time slot. $\vec{P}_{S}$ stands for the compatible pairs and $\vec{P}_{N S}$ for non-simultaneous pairs.

\section{B. Device UEs Pair Scenario}

Here we explain the main procedure of pair analysis. Pair analysis uses beam steering strategy to maximize throughput. As steering beam away reduces signal strength, but improves the pair throughput from reduced interference, UE does not always have to choose the beam that has the most antenna gain.

Steering strategy set are defined by $\bar{K}$. Assuming the sequence of two UEs $i$ and $j$ satisfies $\alpha_{j}>\alpha_{i}$, the design proposes five strategy, represented by $k$ and are follows:

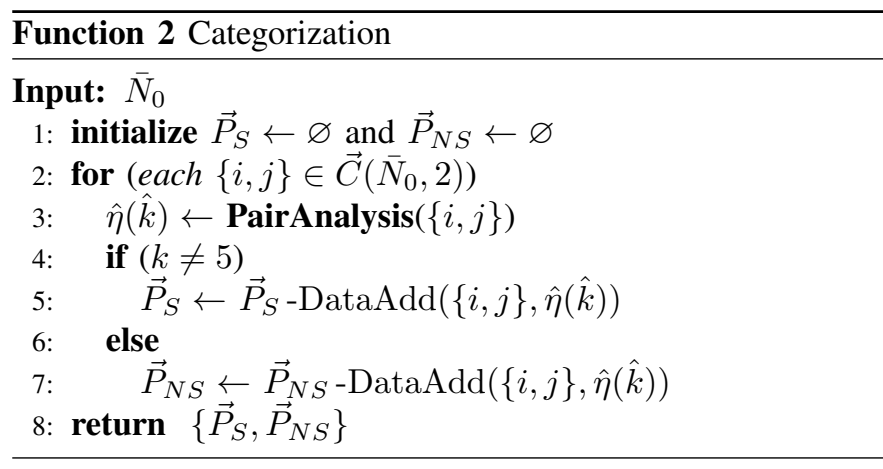

$$
\begin{aligned}
& k=1 \rightarrow\left(\alpha_{i}, \alpha_{j}\right), \text { original beamform } \\
& k=2 \rightarrow\left(\alpha_{i}-\theta_{B}, \alpha_{j}\right), \text { steer down } \\
& k=3 \rightarrow\left(\alpha_{i}, \alpha_{j}+\theta_{B}\right), \text { steer up } \\
& k=4 \rightarrow\left(\alpha_{i}-\theta_{B}, \alpha_{j}+\theta_{B}\right), \text { steer outward } \\
& k=5 \rightarrow\left(\alpha_{i}, \alpha_{j}\right), \text { different time-slot }
\end{aligned}
$$

Steering is not necessary if single UE optimized $\alpha_{i}$ and $\alpha_{j}$ differ by $\theta_{B}$ or more. The interference strength is constant value and steering will only reduce SINR.

Fig. 3 illustrates strategy $k=3$. Beam $\alpha_{j}$ is steered counterclockwise, and $\alpha_{i}$ remaining as it is. Doing so reduces UE $i$ 's interference, thus results in higher aggregate throughput.

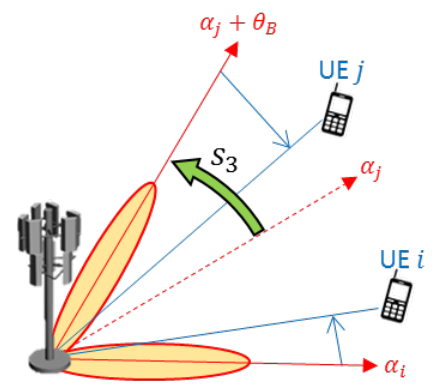

Fig. 3: Example illustration of $k=3$ (steer up)

Selected strategy is determined by SINR, assumed UEs have method to feedback information. Throughput of UE $i$ and $j$ based on responded SINR is $\eta_{i}$ and $\eta_{j}$. Throughput sum is $\eta_{i j}=\eta_{i}+\eta_{j}$.

The pair analysis procedure in Function 3 starts by finding single UE optimized beam, locating the beam with best signal is Beamlocking, returning $\alpha_{i}$ and $\alpha_{j}$ in Line 1-2.

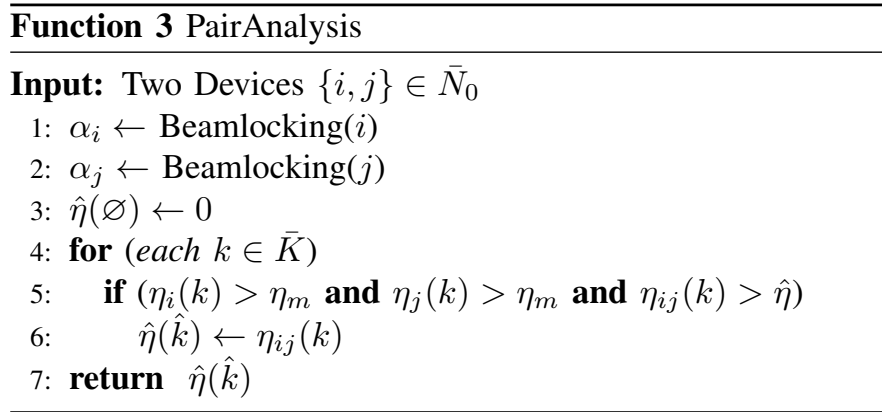

Line 4-6 is where BS iterates through $k \in \bar{K}$ to obtain best steering strategy with pair's highest throughput $\eta_{i}$ and $\eta_{j}$, requiring individual throughput above $\eta_{m}$. 
If strategy $k=\hat{k}$ satisfies minimum throughput requirement and highest throughput $\hat{\eta}(\hat{k})$, then function returns the strategy.

\section{Device UEs Time-Slot Allocation}

With collected information of $\vec{P}_{S}$ and $\vec{P}_{N S}$, Function 4 assign UEs into each time-slot. UEs that can be served concurrently and have higher aggregate throughput will be considered first.

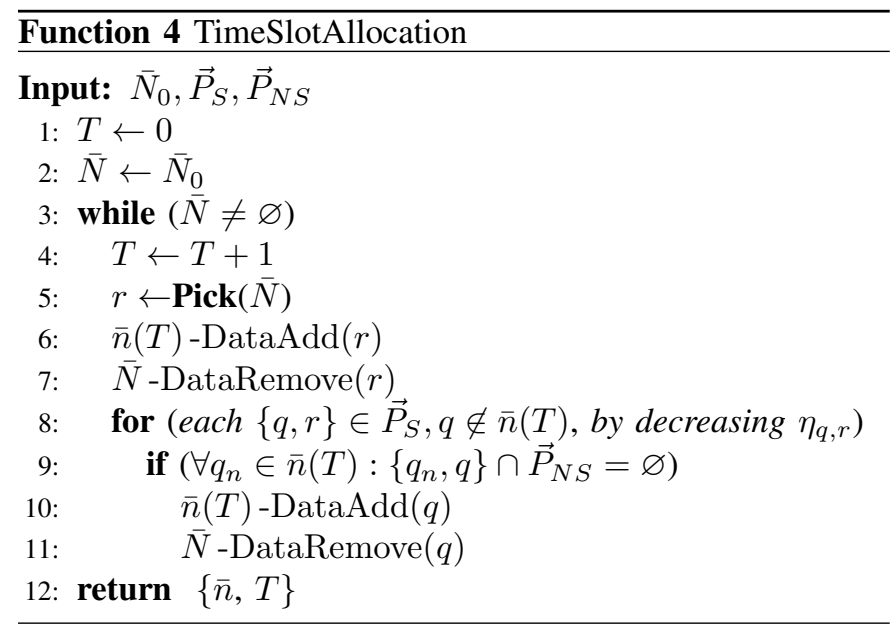

Line 1-2 initializes service time-unit $T$ and unassigned UEs $\bar{N}$. The while loop in Line 3-11 allocates UEs until assignment completion by $\bar{N}=\varnothing$.

A new time unit initialized by Line 4-7, BS approaches by greedy algorithm, from assigning base UE $r$ into $\bar{n}(T)$. UE $r$ from $\operatorname{Pick}(\bar{N})$ is UE with highest throughput in the pair of $\bar{N}$ choose 2 , and the pair is with highest throughput with at least one UE unassigned.

Bottom-Up approach inputs one UE $q$ at a time from categorized pair information. A pair-by-pair checking through all existing combined pair in the time-slot ensures assigned UEs are appropriate for allocation.

Line 8 selected UEs $q$ with base $r$ in $\vec{P}_{S}$, by descending throughput to allocate pairs with higher observed $\eta_{q, r}$ into same time-slot. Line 9 states if any of all combined pair UEs in time-slot does not match with any pairs in $\vec{P}_{N S}$, then allocation algorithm allows $q$ into time-slot.

By repeating through all $\{q, r\} \in \vec{P}_{S}$, continue to next timeslot in Line 4, until completion with $\bar{N}=\varnothing$. Function returns time-slot allocation $\bar{n}$ and $T$.

There is a constraint to upper bound of allowable allocated and Eq. (8) derives $n_{\max }$ by $\min _{i \in \bar{n}(t)} \gamma_{i}$ in time-slot $t$, preventing overflow from excessive power share.

$$
\eta_{i}(\text { Simultaneous })>\eta_{i}(\text { TDMA })
$$

Define $\eta_{i}$ (Simultaneous) the throughput of UE $i$ with other UE $j$ s in $\bar{n}(t)$. Define $\eta_{i}($ TDMA) is UE $i$ served by halving throughput without interference, equivalent two UEs taking individual service. If inequality holds false means the UE has better choice with TDMA instead. As device number exceeds $n_{\max }$, BS will stop allocating and is the termination of the loop. Values of $n_{\max }$ depends on throughput function.

\section{Device UEs Fill-Up Allocation}

With allocation complete and fill-up requested, function re-iterates through time-slots and determines another service opportunity for UEs from ones with minimum throughput from Beamlocking.

Line 1-3 selects UE $q$ unassigned in specified time-slot $\bar{N}_{0} \backslash \bar{n}(t)$ by increasing throughput. Reassign if $q$ combined with UEs in $\bar{n}(t)$ does not match pairs in $\vec{P}_{N S}$.

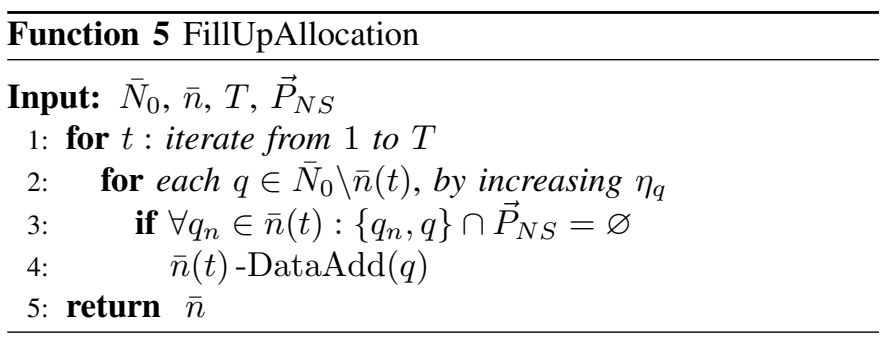

The fill-up process should satisfy the upper bound $n_{\max }$ by inequality of Eq. (8).

\section{E. Device UEs Beam Allocation}

The heuristic algorithm will finalize the spatial resources for UEs in each time-slots by beam set $\bar{\alpha}$.

Line 3 iterates from arbitrary $q$ in time $t$. Line 4-5 finds the UE nearest to $q$, and apply $q$ 's strategy previously recorded $k$ for its beam. Continue to nearest UE until completion of task.

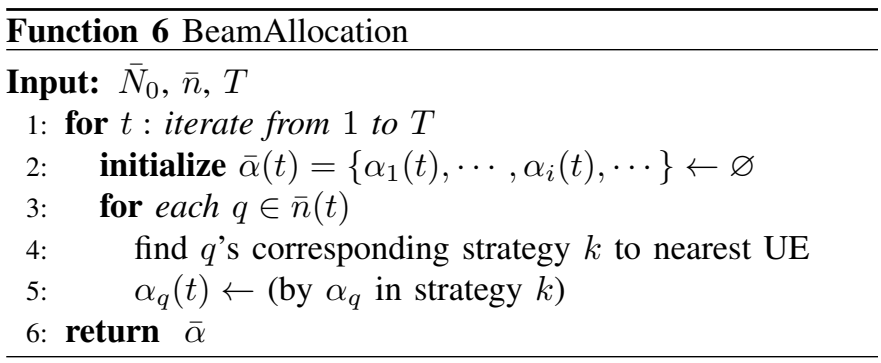

If strategy's resulting beam direction contradicts among pairs, then merely select the original beamlocking beam. For example, UEs $q_{1}, q_{2}$, and $q_{3}$ are in increasing angle sequence and $\alpha_{1}, \alpha_{2}=\alpha_{1}+\theta_{B}$, and $\alpha_{3}=\alpha_{2}+\theta_{B}$. The strategy of $q_{1}$ and $q_{2}$ steers $q_{2}$ toward $\alpha_{3}$, while $q_{3}$ will be assigned with $\alpha_{3}$. In this case, $q_{2}$ will obtain $\alpha_{2}$ instead.

\section{Performance Evaluation}

There are many combinations to put UEs in several time slots to have spatial gain. Exhaustive search can find the best combination that achieves the highest throughput but might not be an efficient option. In this section we compare PWBU algorithm to the results of exhaustive approach in various perspective.

Study of performance via Matlab, channel modelled by official document released by IEEE TGad [15]. The official document portrays characteristic of channel model by reflection decay and attenuation of LoS and NLoS as that of mentioned in III-C. The received SINR value is then mapped to throughput report of [17] for numerical results. 


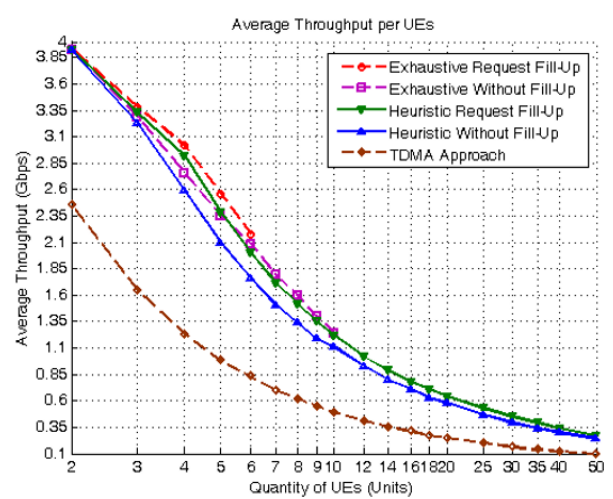

(a) Average Throughput per UEs

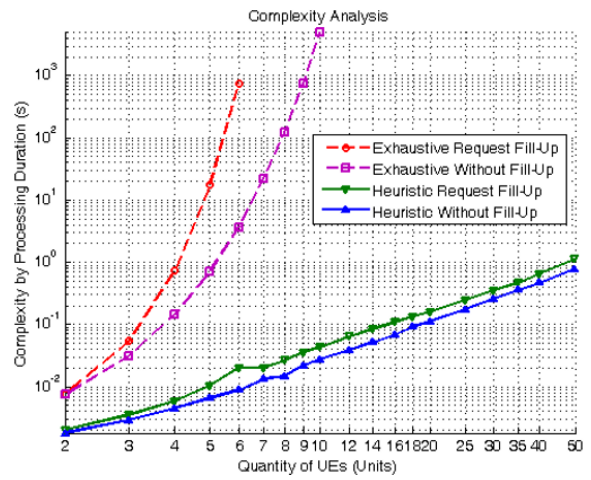

(b) Complexity Analysis

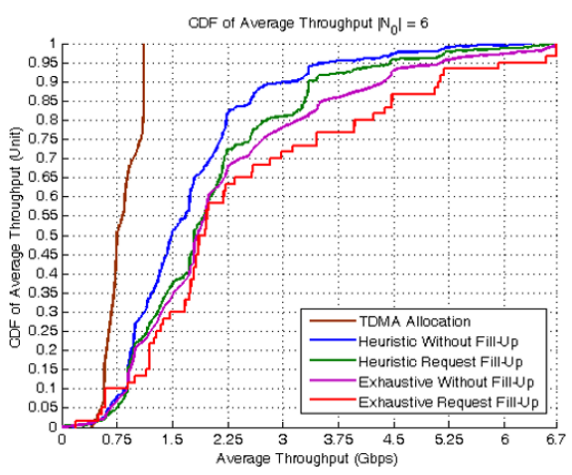

(c) CDF of Average Throughput $\left|\bar{N}_{0}\right|=6$

Fig. 4: Performance Evaluation by Parameters of TABLE I

\section{A. Exhaustive Approach}

Exhaustive approach without fill-up examines all combinations by allocating UEs into each time-slots with different quantity. Each UE is served exactly once. Starting from all UEs simultaneously served until TDMA, serving exactly one UE in every time-slot.

Combination of request fill-up derives from the sequence without fill-up, then generates additional combination by allocating more UEs into these slots.

Exhaustive approach will examine all these combinations and perform deliberate search to find best performance, while heuristic algorithm will complete resource allocation by bottom-up approach.

\section{B. Numerical Results}

Proposed heuristic algorithm will be compared with exhaustive approach and TDMA. The comparison will focus on average and CDF of throughput and complexity. Simulation parameters are listed in Table I.

TABLE I: System Environment parameters

\begin{tabular}{|c|c|}
\hline Frequency $\left(f_{c}\right)$ & $60(\mathrm{GHz})$ \\
\hline Bandwidth $\left(B_{W}\right)$ & $2.16(\mathrm{GHz})$ \\
\hline Noise $\left(G_{W}\right)$ & $-80.6(\mathrm{dBm})$ \\
\hline Tx Power $\left(p_{x, 0}\right)$ & $10(\mathrm{dBm})$ \\
\hline LoS Ray & $1($ Cluster Units) \\
\hline NLoS Ray & $17($ Cluster Units) \\
\hline BS Antenna & Switched Directional, $\theta_{B}=15^{\circ}$ \\
\hline UE(s) Antenna & Omni-Directional \\
\hline Distribution Distance & (uniform) $1(\mathrm{~m})$ to $50(\mathrm{~m})$ \\
\hline Distribution Angle & (uniform) $-180^{\circ}$ to $180^{\circ}$ \\
\hline
\end{tabular}

The average throughput with respect to total amount of associated UEs is in Fig. 4a. Average throughput decreases as UEs increase from 2 to 50 . The performance without or request fill-up both show higher throughput than TDMA. In both of the approaches, exhaustive and PWBU algorithm, request fill-up have higher throughput than without fill-up.

Exhaustive results of system throughput chosen by without and request fill-up are optimal results. Heuristic PWBU are at sub-optimal within $20 \%$ of optimal performance, considering visible exhaustive data. Although at sub-optimal, the allocation shows better time efficiency shown in Fig. 4c.

The complexity is analysed by duration. Designed PWBU algorithm complexity is $T(n) \approx O\left(n^{5}\right)$, fifth degree polynomial. Exhaustive results are in exponential. With exhaustive's complexity, without fill-up has to terminate at 10 UEs and request fill-up at 6 UEs.

Fig. $4 \mathrm{~b}$ is the CDF of throughput without and request fillup, of exhaustive, PWBU, and TDMA with 6 UEs. Exhaustive exhibits average better performance without dispersion of maximum and minimum throughput. PWBU algorithm shows sub-optimal with less difference between maximum and minimum throughput. The designed PWBU algorithm has shown improvements to TDMA approach and sub-optimal with timeefficiency to exhaustive ones.

\section{CONCLUSION}

This paper targets time-spatial resource allocation for higher throughput by Pair-Wisely Bottom-Up (PWBU) algorithm, with basic and switched antenna added with adaptive smart antenna capable of simultaneous beamforming.

Sharing of transmitted power to UEs, from simultaneous beamforming, reduces throughput at the first glance. However, reduced waiting time for UEs serves as resource compensation and will leverage throughput in long terms. Steering of beam directions also shows reduced amount of interferences. The proposed PWBU algorithm is a potential approach to increase throughput performance under the beamforming assumption we made.

\section{ACKNOWLEDGEMENTS}

This work was also supported by Ministry of Science and Technology, National Taiwan University and Media Tek Inc. under Grants MOST 103-2622-E-002-034.

\section{REFERENCES}

[1] A. Swindlehurst, E. Ayanoglu, P. Heydari, and F. Capolino, "Millimeter-Wave Massive MIMO: the Next Wireless Revolution," Communications Magazine, IEEE, September 2014. 
[2] Y. Wang, J. Zhang, J. Wang, and B. Bensaou, "Coordinated Fair Resource Sharing In Dense Indoor Wireless Networks," in 2014 IFIP Networking Conference, June 2014, pp. 1-9.

[3] I. Jawhar and J. Wu, "Resource Allocation in Wireless Networks Using Directional Antennas," in Fourth Annual IEEE International Conference on Pervasive Computing and Communications, 2006. PerCom 2006., March 2006, pp. 10 pp.-327.

[4] L. Bao and J. Garcia-Luna-Aceves, "Transmission Scheduling in Ad Hoc Networks with Directional Antennas," in Proceedings of the 8th Annual International Conference on Mobile Computing and Networking, ser. MobiCom '02. New York, NY, USA: ACM, 2002, pp. 48-58.

[5] D. Lal, R. Toshniwal, R. Radhakrishnan, D. Agrawal, and J. Caffery, "A Novel MAC Layer Protocol for Space Division Multiple Access in Wireless ad hoc Networks," in Eleventh International Conference on Computer Communications and Networks, 2002. Proceedings., Oct 2002, pp. 614-619.

[6] T. Rappaport, S. Sun, R. Mayzus, H. Zhao, Y. Azar, K. Wang, G. Wong, J. Schulz, M. Samimi, and F. Gutierrez, "Millimeter Wave Mobile Communications for 5G Cellular: It Will Work!" IEEE Access, vol. 1, pp. 335-349, 2013.

[7] M. Akdeniz, Y. Liu, M. Samimi, S. Sun, S. Rangan, T. Rappaport, and E. Erkip, "Millimeter Wave Channel Modeling and Cellular Capacity Evaluation," IEEE Journal on Selected Areas in Communications, vol. 32, no. 6, pp. 1164-1179, June 2014.

[8] S. Sun, G. MacCartney, M. Samimi, S. Nie, and T. Rappaport, "Millimeter Wave Multi-Beam Antenna Combining For 5G Cellular Link Improvement in New York City," in 2014 IEEE International Conference on Communications (ICC), June 2014, pp. 5468-5473.

[9] I. K. Son, S. Mao, M. Gong, and Y. Li, "On Frame-based Scheduling for Directional mmWave WPANs," in 2012 Proceedings IEEE INFOCOM, March 2012, pp. 2149-2157.

[10] Y. K. Meejoung Kim and W. Lee, "Resource Allocation Scheme for Millimeter Wave-Based WPANs Using Directional Antennas," ETRI Journal, vol. 36, no. 3, pp. 385-395, June 2014.

[11] H.-N. Dai, K.-W. Ng, M. Li, and M.-Y. Wu, "An overview of using directional antennas in wireless networks," International Journal of Communication Systems, vol. 26, no. 4, pp. 413-448, April 2013.

[12] M. Gong, D. Akhmetov, R. Want, and S. Mao, "Multi-User Operation in mmWave Wireless Networks," in 2011 IEEE International Conference on Communications (ICC), June 2011, pp. 1-6.

[13] M. Gong, D. Akhmetov, and R. Want, "Directional CSMA/CA Protocol with Spatial Reuse for mmWave Wireless Networks," in 2010 IEEE Global Telecommunications Conference (GLOBECOM 2010), Dec 2010, pp. 1-5.

[14] S. Kasiviswanathan, B. Zhao, S. Vasudevan, and B. Urgaonkar, "Bandwidth Provisioning in Infrastructure-based Wireless Networks Employing Directional Antennas ," Pervasive and Mobile Computing, vol. 7, no. 1, pp. $114-127,2011$.

[15] A. Maltsev, V. Erceg, E. Perahia, C. Hansen, R. Maslennikov, A. Lomayev, A. Sevastyanov, A. Khoryaev, G. Morozov, M. Jacob, S. Priebe, T. Krner, S. Kato, H. Sawada, K. Sato, and H. Harada, "Channel Models for $60 \mathrm{GHz}$ WLAN Systems," May 2010.

[16] E. Perahia, C. Cordeiro, M. Park, and L. Yang, "IEEE 802.11ad: Defining the Next Generation Multi-Gbps Wi-Fi," in Consumer Communications and Networking Conference (CCNC), 2010 7th IEEE, Jan 2010, pp. 1-5.

[17] X. Zhu, A. Doufexi, and T. Kocak, "Throughput and Coverage Performance for IEEE 802.11ad Millimeter-Wave WPANs," in 2011 IEEE 73rd Vehicular Technology Conference (VTC Spring), May 2011, pp. 1-5. 